\title{
On Optimization of Power Coefficient of HAWT
}

\author{
Marat Z. Dosaev1, Lyubov A. Klimina1, Boris Ya. Lokshin1, Yury D. Selyutskiy1, \\ Shih-Shin Hwang ${ }^{2}$ \\ ${ }^{1}$ Institute of Mechanics, Lomonosov Moscow State University, Moscow, Russia \\ ${ }^{2}$ Mechanical Engineering Department, Chien Hsin University of Science and Technologies, Zhongli City, Taiwan \\ Email: dosayev@imec.msu.ru
}

Received December 2013

\begin{abstract}
The horizontal axis wind turbine (HAWT) blades rotation in the steady wind flow is considered. We discuss the problem of determining the blade twist which could guarantee the maximum value of the power coefficient. We define the blade twist as the technological turn of sections of blade around its axis. This turn changes the effective pitch angle of turbine blade along its length. For description of aerodynamic load upon the blades we used the quasi-steady approach. Air velocities of centers of pressure of blade sections are represented when taking into account components induced by flow and vortex. We reduced the functional maximization problem to find the maximum of non-dimensional function. This function is given by Riemann integral depending on section pitch angle and tip speed ratio. We suggested the algorithm for solving the problem under consideration for a given blade shape.
\end{abstract}

\section{Keywords}

HAWT Blade; Tip Speed Ratio; Maximum of Power Coefficient

\section{Introduction}

The problem of designing the new shape for wind turbine blade is very complicated. Along with taking into account vibration and strength properties of blade, one must allow for necessity to utilize the maximum energy of wind. Wide spreading of different types of wind turbine enables to construct engineering techniques for blade design. For example, in $[1,2]$ authors proposed the engineering method for choosing blades parameters, such as the aerodynamic profile, blade width, pitch angle, and etc for several sections along the flow. On the other hand the wind turbine aerodynamics is comprehensively studied [3]. Some authors use analytical approaches for power efficiency estimating of wind turbine. The method based on using Goldstein functions is proposed in [4] for ideal turbine with the finite number of blades. The simple enough algorithm of numerical estimation of the upper bound of the power coefficient is suggested.

In present paper, we propose the analytical-computational approach for calculating the distribution of twist blade angle along the blade length, which guarantee the maximum value of power coefficient for given blade section profile and give distribution of blade width (Figure 1). We use the mechanical-mathematical model of 


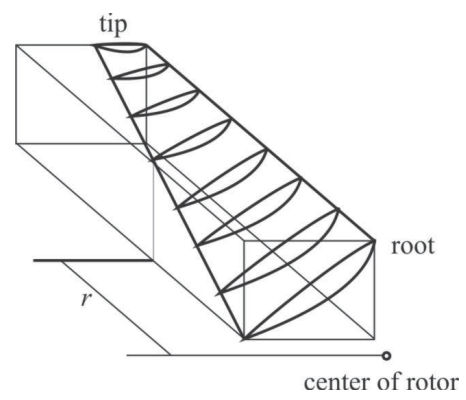

Figure 1. Blade twist for several sections.

aerodynamic load upon turbine blades developed in [5]. This model is based on quasi-steady approach. In order to advance this model we introduce the components that related with induced velocities (see for example, [5]).

\section{Problem Formulation}

We consider the turbine with radius $R$ (where $R$ is a distance between axis of rotation and blade tip) that has $n$ blades (usually $n=3-6$ ) and rotates with angular speed $\Omega$ in steady airflow. The wind speed $W$ is directed along the turbine axis. The output power $P$ of turbine looks as following:

$$
P=0.5 C_{p}(\lambda) \cdot \rho S W^{3},
$$

where $\lambda$ is TSR (tip speed ratio) of turbine, $\rho$ is the air density, $S=\pi R^{2}$ is turbine swept area, $C_{p}(\lambda)$ is the power coefficient that characterizes the efficiency of wind turbine.

We assume that the blade section, which is located at distance $r(0<r<R)$ from axis of rotation, represents the given airfoil. The shape of section is the same for all blade sections. We formulate the following problem: to determine the specified value $\lambda$ and specified dependence $\vartheta=\vartheta(r)$ for twist angle of blade along its length, which ensure the maximum value of power coefficient.

We use the flat cross-section hypothesis for needed characteristics obtaining. We allocate the section element $d r$ which is located at distance $r$ from axis of rotation (Figure 2). Dependence $b=b(r)$ is given. The air velocity of point of intersection of blade axis and blade section is a sum of flow speed $W(1-a)$ and speed $r \Omega\left(1+a^{\prime}\right)$ of its rotation. Here coefficients $a, a^{\prime}$ to be determined are functions of $r$.

In Figure 3, the side view of this element is shown. In this projection, it is convenient to introduce all required values: pitch angle $\theta$, angle of attack $\alpha$, velocity $\mathbf{V}$ with respect to the flow, angle $\phi$ between the turbine plane and $\mathbf{V}$, and vectors $\mathbf{D}, \mathbf{L}$-components of the aerodynamic force (drag and lift, respectively) acting upon the selected element. By construction, we have:

$$
\begin{aligned}
& \theta=\phi-\alpha, \operatorname{tg} \phi=W(1-a) / r \Omega\left(1+a^{\prime}\right) \\
& W(1-a)=V \sin \phi, r \Omega\left(1+a^{\prime}\right)=V \cos \phi, \\
& V=\sqrt{W^{2}(1-a)^{2}+(r \Omega)^{2}\left(1+a^{\prime}\right)^{2}}
\end{aligned}
$$

Aerodynamic forces are defined by the following conventional formulae:

$$
D=0.5 C_{D}(\alpha) \rho V^{2} d S, L=0.5 C_{L}(\alpha) \rho V^{2} d S
$$

where $d S=b(r) d r$ is the element area, and aerodynamic coefficients $C_{D}(\alpha), C_{L}(\alpha)$ are known functions for a given airfoil [6]. Note that such approach to describing the aerodynamics was used in [7].

The aerodynamic torque $d M$ with respect to the rotation axis produced on this element is given by the expression

$$
d M=r(L \cos \phi-D \sin \phi)
$$

or, taking into account (2.1) and (2.2),

$$
\begin{aligned}
d M & =0.5 r b(r)\left(C_{L}(\alpha) \cos \phi-C_{D}(\alpha) \sin \phi\right) \rho V^{2} d r \\
& =0.5 r b(r) \rho W^{2}\left[(1-a) C_{L}(\alpha)-\left(1+a^{\prime}\right) C_{D}(\alpha) r \Omega / W\right] \times \sqrt{(1-a)^{2}+\left(r \Omega\left(1+a^{\prime}\right) / W\right)^{2}} d r
\end{aligned}
$$




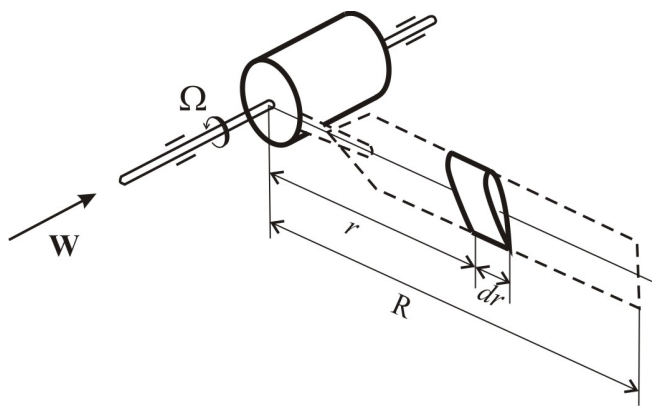

Figure 2. Blade element.

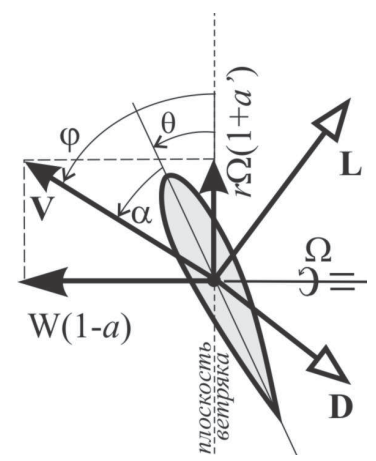

Figure 3. Side view of blade element.

Denote:

$$
\omega=r \Omega / W=\lambda r / R
$$

Then the torque produced by all blades can be represented as an integral

$$
M=\int_{0}^{R} 0.5 n \rho W^{2} r b(r)\left[(1-a) C_{L}(\alpha)-\left(1+a^{\prime}\right) C_{D}(\alpha) \omega\right] \sqrt{(1-a)^{2}+\omega^{2}\left(1+a^{\prime}\right)^{2}} d r
$$

The power $P=\Omega M$ looks as follows:

$$
P=0.5 n \rho W^{3} \lambda \int_{0}^{R} r b(r) R^{-1}\left[(1-a) C_{L}(\alpha)-\left(1+a^{\prime}\right) C_{D}(\alpha) \omega\right] \sqrt{(1-a)^{2}+\omega^{2}\left(1+a^{\prime}\right)^{2}} d r
$$

Substituting $r$ in (2.4) using the expression (2.3), we obtain

$$
P=0.5 n \rho R^{2} W^{3} \Phi(\lambda)
$$

Here the non-dimensional function is introduced

$$
\Phi(\lambda)=\lambda^{-1} \int_{0}^{\lambda} \omega b R^{-1}\left[(1-a) C_{L}(\alpha)-\left(1+a^{\prime}\right) C_{D}(\alpha) \omega\right] \sqrt{(1-a)^{2}+\omega^{2}\left(1+a^{\prime}\right)^{2}} d \omega
$$

Comparing (2.5) with the general formula $P=0.5 C_{p} \rho \pi R^{2} W^{3}$ for the power produced by the wind turbine, one can readily see that the following relation holds:

$$
C_{p}(\lambda)=n \pi^{-1} \Phi(\lambda)
$$

and the initial optimization problem is reduced to the problem of maximum search for the function (2.6).

\section{Solution of the Problem}

Before solving the problem of optimization of this coefficient (values to be varied are $\theta(r)$ and $\lambda$, blade chord $b$ is a given function of $r=\omega R / \lambda$, values $a, a^{\prime}$ are also to be defined as functions of $r=\omega R / \lambda$ ), consider the process of calculation of the integrand in (2.6) for fixed $r, \omega, \lambda, b, \theta$. For that, following [3], we 
introduce the adjustment coefficient $F=2 \arccos \left(e^{-f}\right) / \pi$, taking into account losses at the blade tip, where $f=n(\lambda-\omega) / 2 \omega, \quad \sigma=n b \lambda(2 \pi R \omega)^{-1}, \quad C_{N}=C_{L}(\alpha) \cos \phi+C_{D}(\alpha) \sin \phi$ is the component of aerodynamic force along HAWT the axis, and $C_{T}=C_{L}(\alpha) \sin \phi-C_{D}(\alpha) \cos \phi$ is the component of aerodynamic force lying in the turbine plane. Then induced speeds coefficients can be represented as follows [3]:

$$
\begin{aligned}
& a=\sigma C_{N}(\alpha)\left(4 F \sin ^{2} \varphi+\sigma C_{N}(\alpha)\right)^{-1}, \\
& a^{\prime}=\sigma C_{T}(\alpha)\left(4 F \sin \varphi \cos \varphi-\sigma C_{T}(\alpha)\right)^{-1}
\end{aligned}
$$

Angles $\alpha$ and $\varphi$ can be expressed as follows (using (2.1) and (2.3)):

$$
\alpha=\varphi-\theta, \varphi=\operatorname{arctg}\left((1-a) \omega^{-1}\left(1+a^{\prime-1}\right)\right)
$$

Relations (3.1)-(3.2) make a system of four equations with respect to $a, a^{\prime}, \varphi, \alpha$. Contrarily to the iterative method of solving these equations proposed in [3,5], consider another way for determining the sought values. Substituting (3.1) into the second Equation (3.2), we obtain

$$
4 F\left(\omega \sin ^{2} \varphi-\sin \varphi \cos \varphi\right)+\sigma\left(\omega C_{N}+C_{T}\right)=0
$$

From here, $\varphi$ is expressed via $\alpha, \omega$, $\lambda$. In particular, for $F \approx 0(\omega \approx \lambda)$ we have: $\varphi=-\operatorname{arctg}\left[\left(\lambda C_{L}-C_{D}\right) /\left(\lambda C_{D}+C_{L}\right)\right]$. After having solved (3.3), parameters $a, a^{\prime}$ are also expressed from (3.1) via $\alpha, \omega, \lambda$, and then the angle $\theta$ is determined from the first Equation (3.2) as a function of the same parameters $\alpha, \omega, \lambda$. Thus, the integrand in (2.6) represents an explicit function of $\alpha, \omega, \lambda$. Such representation is more preferable for solving the original problem of maximization of the coefficient $C_{p}$.

Now return to the problem of maximization of $C_{p}$, or of function $\Phi(\lambda)$. Taking into account that $\lambda$ is a constant (though unknown), the integral (2.6) can be interpreted as a functional defined on the class of differentiable functions $\alpha(\omega)$. Then the source problem is deduced to the problem of choice of a function $\alpha=\alpha^{0}(\omega, \lambda)$ that delivers maximum to the integral, and of subsequent choice of such a value $\lambda^{0}$ that delivers maximum value to the function $\Phi(\lambda)$.

In order to find $\alpha^{0}(\omega)$, we use the convenient method for solving variational problems, we obtain the following necessary condition of the maximum of the integral (Euler-Lagrange equation): the full derivative of the integrand with respect to $\alpha$ is zero (this equation is not written here due to its complexity).

In [5], results of numerical solution of Equations (3.1)-(3.2) are given for several NACA airfoils, from which one can see that coefficients $a, a^{\prime}$ almost do not change for $\omega>1$. Hence, we assume (as the first approximation) that

$$
a=\text { const }, \quad a^{\prime}=\text { const }
$$

Then the mentioned Euler-Lagrange equation looks as follows

$$
(1-a) d C_{L} / d \alpha-\omega\left(1+a^{\prime}\right) d C_{D} / d \alpha=0
$$

From (3.5) it is possible to find $\alpha$ as a function of $\omega: \alpha=\alpha(\omega)$. One of branches of this solution will contain the optimal dependence $\alpha=\alpha^{0}(\omega, \lambda)$, and from (3.3) (or from (3.2)) it is possible to determine the intermediate variable $\varphi$, and then from (3.2) it is possible to derive the expression for the desired blade twist containing the undetermined (so far) parameter $\lambda$ :

$$
\theta^{0}(\omega, \lambda)=\operatorname{arctg}\left((1-a)\left(1+a^{\prime-1}\right) \omega^{-1}\right)-\alpha^{0}(\omega, \lambda)
$$

Substituting the obtained expression for $\alpha^{0}(\omega)$ into the integrand in (2.6), we obtain the function $\Phi(\lambda)$ of single argument $\lambda$, maximum value of which is determined analytically (if possible) or numerically. Thus, the desired optimal value $\lambda^{0}$ will be found, after which the optimal blade twist is determined from (3.6) as a function of $\omega,\left(\omega \in\left(0, \lambda^{0}\right]\right)$. Note that $\omega=\lambda^{0} r / R,(r \in(0, R])$.

In principle, the problem is solved.

Taking into account dependences $C_{L}(\alpha), C_{D}(\alpha)$ (e.g., [6]), one can show that the solution $\alpha=\alpha(\omega)$ of Equation (3.5) exists as a monotonically decaying function. If it delivers maximum to the functional (3.6), then upon having determined the optimal value of $\lambda^{0}$ and returning back to the variable $r$, the optimal blade twist can also be decreasing function $\theta^{0}(r)=\operatorname{arctg}\left(\left(R / \lambda^{0} r\right)(1-a)\left(1+a^{\prime}\right)^{-1}\right)-\alpha^{0}\left(\lambda^{0} r / R\right)$. 
This monotony is qualitatively confirmed by the practice of HAWT design.

\section{Conclusion}

In this paper we studied the problem of maximization of power coefficient for a HAWT by optimization of a blade twist and tip speed ratio. We obtained an algorithm for determining of the optimal solution of this problem in the frame of the quasi-steady model of aerodynamic action taking into account induced velocities. Each step of this algorithm depends on parameters of the model, so the final shape of the twist can be specified for each particular airfoil. Still qualitative features of the optimal solution remain for the general case: for instance, there exists the optimal pitch angle function that is monotone along the blade length.

\section{Acknowledgements}

The work is partially supported by RFBR, projects NN 11-08-92005, 12-01-00364, and 14-08-01130.

\section{References}

[1] Perli, S.B. (1938) Windpump and Windelectrical Units. ONTI, Kharkov. (in Russian)

[2] Sabinin, G.H. (1931) Theory and Aerodynamic Calculation of Wind Engines. Trudi TsAGI, Moscow. (in Russian)

[3] Hansen, M.O.L. (2000) Aerodynamics of Wind Turbines. James and James, London.

[4] Okulov, V.L. and Sorensen, G.N. (2008) Ideal Wind Turbine with Finite Number of Blades. DAN, 420, 478-483. (in Russian)

[5] Tenguria, N., Mittal, N.D. and Ahmed, S. (2011) Evaluation of Performance of Horizontal Axis Wind Turbine Blades Based on Optimal Rotor Theory. Journal of Urban and Environmental Engineering, 5, 15-23. http://dx.doi.org/10.4090/juee.2011.v5n1.015023

[6] Kashafutdinov, S.T. and Lushin, V.N. (1994) Atlas of Aerodynamic Characteristics of Wing Airfoils. SO RASHN, Novosibirsk. (in Russian)

[7] Dosaev, M.Z., Kobrin, A.I., Lokshin, B.Ya., Samsonov, V.A. and Selyutskiy, Yu.D. (2007) Constructive Theory of Small-Scale Power Generators. Part I, MSU Publishing, Moscow. 